\title{
Relevamiento del patrimonio histórico rural mediante fotogrametría (SfM-MVS) en la región pampeana argentina. "Primera Estancia" de Magdalena (Buenos Aires, Argentina)
}

\section{Soledad García Lerena}

(iD) https://orcid.org/0000-0002-8592-1049

Laboratorio de Análisis Cerámico (LAC), Facultad de Ciencias Naturales y Museo, Universidad Nacional de La Plata (UNLP). Calle 64 nº 3 (CP B1900FWA), La Plata, Buenos Aires, Argentina. E-mail: soledad.garcia.lerena@gmail.com

\section{Luciano López}

(iD) http://orcid.org/0000-0003-4072-3273

Instituto de Recursos Minerales (INREMI), Facultad de Ciencias Naturales y Museo, Universidad Nacional de La Plata (UNLP) - Consejo Nacional de Investigaciones Científicas y Técnicas (CONICET). Calle $64 \mathrm{n}^{\circ} 3$ (CP B1900FWA), La Plata, Buenos Aires, Argentina. E-mail: lopezluciano@hotmail.com

\begin{abstract}
Resumen
En el marco del relevamiento del patrimonio histórico rural del partido de Magdalena, se presenta un modelo virtual 3D de una estructura habitacional utilizada como vivienda de los peones de una estancia ganadera generado por Structure from Motion MultiView Stereo (SfM-MVS). Dicha estructura se ubica en la región costera de la provincia de Buenos Aires, zona que cuenta con un importante patrimonio cultural y natural. El flujo de trabajo incluyó la adquisición y procesamiento de fotografías con el objetivo de generar un modelo 3D. Este modelo produce un resultado fotorrealista, sobre el que realizan una serie de visualizaciones y análisis; como el registro de patologías, la obtención de mediciones en gabinete y la generacion de un registro para evaluar el estado de conservación. Se pone en consideración la potencialidad de la técnica frente a la posibilidad de pérdida del patrimonio local. Las fortalezas de esta técnica radican en la reducción del tiempo de la toma de datos, el relevamiento in situ, no destructivo y de bajo costo. Permite obtener reconstrucciones con gran detalle y precisión, útiles para la investigación y la divulgación, lo cual contribuye a la salvaguarda y socialización de los bienes patrimoniales.
\end{abstract}




\title{
Photogrammetry (SfM-MVS) survey of rural historical heritage in the Pampean region. The "Primera Estancia”, Magdalena, Buenos Aires, Argentina
}

\begin{abstract}
We present a 3D virtual model generated with Structure from Motion Multi-View Stereo (SfM-MVS) of a house, formerly used as a dwelling by farmworkers, located in the cattle ranch named "Primera Estancia". The ranch, a private property located in the coastal area of Buenos Aires province, has an important cultural and natural heritage. The survey included the acquisition and processing of photographs to generate a 3D model. This model produces a photorealistic result, on which a series of visualizations and analyses can be performed: recording of pathologies, obtaining of measurements in the laboratory, and generation of a record to evaluate the preservation state. We highlight the potential of this technique in light of the loss of local heritage. It also has advantages such as the reduction of the time and effort in data collection with an in situ, non-destructive, and low-cost technique. This technique also allows obtaining reconstructions with detail and precision, useful for research and science communication. It is a very important tool for the socialization of knowledge, and dissemination of the local heritage.
\end{abstract}

KEYWORDS: Structure from motion; Heritage; 3D reconstruction; Parque Costero del Sur

\section{Introducción}

Con el fin de proteger la región costera de los partidos de Magdalena y Punta Indio en la provincia de Buenos Aires (Argentina), la UNESCO declaró en el año 1984 el "Parque Costero del Sur" (PCS) como Reserva Mundial de Biosfera Natural y Cultural por su valioso patrimonio natural y cultural. Constituye una franja costera de cinco $\mathrm{km}$ de ancho por $70 \mathrm{~km}$ de largo, que se inicia inmediatamente después del predio del Regimiento Octavo de Infantería en el partido de Magdalena y finaliza en la intersección de la Ruta Provincial No 11 y la Ruta Provincial No 36, partido de Punta Indio (Paleo et al., 2015; Figura 1). En este sector se distinguen diversos ambientes que son hábitat de numerosas especies animales, entre las que se destaca la biodiversidad de aves, reptiles y mamíferos. Asimismo, el PCS configura un paisaje antrópico que es resultado de la prolongada ocupación humana de la zona. De esta manera, muchos elementos del territorio concebidos como "naturales" constituyen en realidad el resultado de la interacción entre los seres humanos y su entorno a través del tiempo. El territorio de la reserva se encuentra, en su mayoría, integrado por campos y estancias privadas dedicados principalmente a la ganadería o a la extracción de material calcáreo del subsuelo (Paleo, García Lerena, Stampella, Doumecq y Pochettino, 2016). Esta región fue ocupada por poblaciones hispanocriollas de manera temprana por lo cual en su extensión se conservan registros materiales de diferentes temporalidades, los que no están incluidos en su totalidad en el área del PCS. En la actualidad, se está tramitando en la UNESCO la ampliación de su extensión para incluir otros sectores con valor patrimonial, en particular hacia el norte de los límites actuales (Figura 1).

En los últimos años, se han realizado relevamientos de los sitios arquitectónicos de valor patrimonial, en su mayoría pertenecientes a estancias ganaderas cuyo auge tuvo lugar en las últimas décadas del siglo XIX y las primeras del siglo XX. Vestigios de este momento, se conservan a lo largo del sector costero de los partidos de Magdalena y Punta Indio numerosas construcciones pertenecientes a estas estancias. En la zona conviven cascos con gran cantidad de dependencias, imponentes casas principales y jardines diseñados 


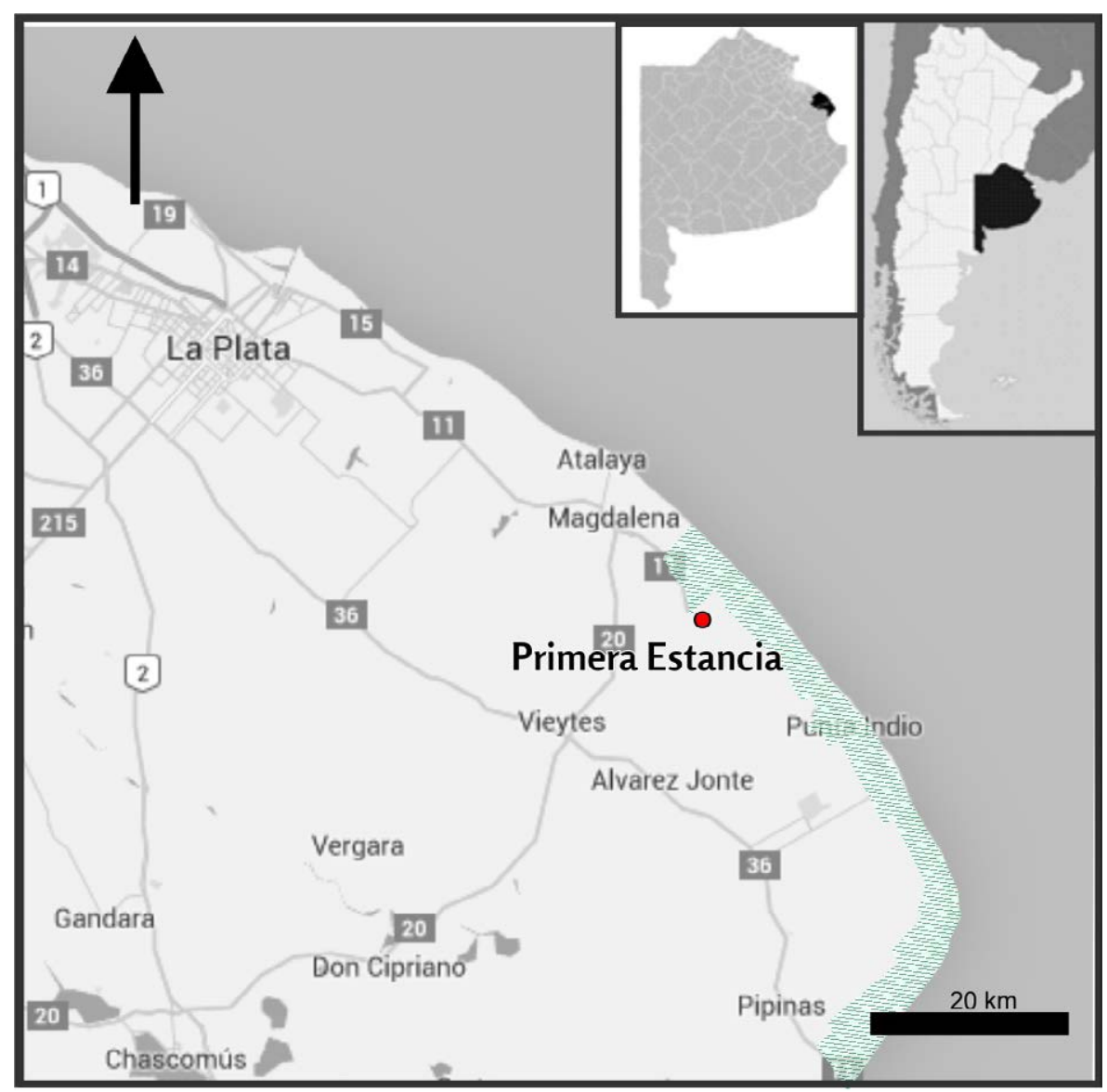

Figura 1. Ubicación del Parque Costero del Sur (sombreado verde) y la Primera Estancia, en la provincia de Buenos Aires, Argentina.

en algunos casos por el paisajista Carlos Thays, con pequeñas estancias y sus modestos "ranchos", relictos de la diversidad del pasado local. El abordaje de distintos sectores sociales, tanto de los grandes propietarios terratenientes y sus familias como de los trabajadores de las estancias, permite conocer la vida cotidiana en el pasado, los cambios y continuidades en las prácticas y representaciones, y cómo el pasado es significado por la comunidad actual. La arqueología, a partir del estudio de la materialidad y la espacialidad en el pasado, constituye una herramienta privilegiada para ello.

Este importante patrimonio histórico rural perteneciente a estancias ganaderas de la zona, como mencionamos, se encuentra mayoritariamente en propiedades privadas. Muchos propietarios no habitan en las estancias en forma habitual, las cuales están a cargo de mayordomos o encargados que, en general, no están autorizados para dejar ingresar a personas ajenas. En otros casos, los propios dueños explícitamente, ya sea por temor o por otras situaciones conflictivas, no desean el ingreso de particulares en sus propiedades. En otras ocasiones, las escasas posibilidades económicas que tienen algunos propietarios para el mantenimiento y puesta en valor de elementos patrimoniales, se conjugan con el desinterés y falta de acciones concretas de los representantes de organismos gubernamentales, lo cual genera pocas posibilidades de que determinadas estructuras perduren en el tiempo. Estas situaciones traen aparejadas una serie de dificultades para la accesibilidad y posibilidades para su estudio y puesta en valor, y en particular para la socialización y apropiación de los elementos patrimoniales para el resto de la comunidad. En general, la experiencia nos ha enseñado que luego de explicar nuestra pertenencia institucional y nuestros objetivos de trabajo, somos autorizados para 
realizar tareas de investigación, en algunos casos abiertamente y en otros con reticencia. Sin embargo, sostenemos que el trabajo conjunto con la comunidad y la participación activa de los pobladores en la recuperación, puesta en valor y socialización de aspectos de su patrimonio, es decir con su patrimonialización, facilita generar una apropiación colectiva del mismo, contribuyendo al fortalecimiento y resignificación de la identidad y pertenencia local (Paleo et al., 2015).

En este trabajo se expone un modelo virtual 3D generado por Structure from Motion MultiView Stereo (SfM-MVS) y se analiza su utilidad para el abordaje del patrimonio histórico rural, en particular de construcciones que se encuentran en propiedades privadas. Este tipo de reconstrucciones constituye una herramienta útil de investigación, y también es una forma de contribuir a su salvaguarda y socialización con la comunidad mediante la divulgación (Hatzopoulos et al., 2017; Sánchez Chacón y Shimabukuro Shimabukuro, 2015). De esta manera, se analiza el caso de Primera Estancia, que constituye por sus particularidades un significativo ejemplo de patrimonio histórico rural en estancias privadas y se evalúa su potencial para abordar otras estancias de la zona.

La utilización de SfM-MVS permite el relevamiento de información tridimensional en múltiples escalas de trabajo: se utiliza para el registro de sitios arqueológicos y durante el proceso de excavación (Aparicio Resco, Carmona Barrero, Fernández Díaz y Martín Serrano, 2014; Benavides López et al., 2016; Charquero Ballester, 2016; De Reu et al., 2013, 2014; Verhoeven, Doneus, Briese y Vermeulen, 2012; Willis, Koenig, Black y Castaneda, 2016); a nivel materiales, colecciones y confección de atlas (Eving et al., 2016; Scopigno et al., 2011); incluso a microescala, para el estudio de marcas de corte en materiales óseos (Lorenzo, López y Flores, 2016; Maté González, Yravedra, González-Aguilera, Palomeque-González y Domínguez-Rodrigo, 2015). La generación de información 3D tiene una reciente incidencia en el mundo del patrimonio cultural (Pierdicca, Frontoni, Malinverni, Colosi y Orazi, 2016; Vázquez, y Díaz Pais, 2014). Debido a la especial naturaleza de los objetos o elementos que se inscriben dentro de este tipo de patrimonio (fragilidad, alto grado de deterioro, alta sensibilidad a agentes extraños, tamaño, entre otros aspectos), a la hora de realizar un trabajo de documentación es deseable utilizar tecnologías que sean no invasivas ni destructivas (Díaz Gómez, Jiménez Peiró, Barreda Benavent, Asensi Recuenco y Hervás, 2015).

La reconstrucción 3D por medios digitales tiene múltiples usos en arqueología. En particular, en el campo de la arqueología histórica en la región pampeana argentina tiene un desarrollo incipiente y existen escasos antecedentes de su aplicación en problemáticas vinculadas al patrimonio histórico rural (Vázquez y Díaz Pais, 2014). Los beneficios que aporta esta técnica para la investigación y la divulgación del patrimonio histórico son variados (Hatzopoulos et al., 2017; Pereira Uzal, 2013). La fotogrametría comienza a ser utilizada con mayor asiduidad en arqueología y proporciona de forma semi-automatizada una información con alta calidad, precisa, rápida, objetiva y sujeta a reelaboraciones posteriores (Charquero Ballester, 2016; De Reu et al., 2014). De esta forma, se cuenta con una técnica de bajo costo, que no requiere de equipamiento especializado y que está al alcance de cualquier proyecto o investigador (ver figura 2.7 en Carrivick, Smith y Quincey, 2016). Otras técnicas, como la tecnología láser (LIDAR) o luz estructurada, requieren equipamientos costosos y personal especializado, inaccesibles para muchos profesionales por los costes de su adquisición, a la vez que presentan un manejo complejo (Pereira Uzal, 2013).

\section{Primera Estancia (partido de Magdalena, Buenos Aires)}

Al sur de la ciudad de Magdalena (provincia de Buenos Aires; Figura 1) se conformó, desde mediados del siglo XVIII, una estancia de importantes dimensiones que fue 
denominada "Primera Estancia", propiedad del linaje del estanciero Januario Fernández. Para la segunda mitad del siglo XIX, este establecimiento contaba con una edificación principal y 14 puestos de estancia en donde residían los puesteros o pastores. En la actualidad, el sector del casco de "Primera Estancia", ocupa una superficie de 150 por $100 \mathrm{~m}$ aproximadamente, en donde se localizan estructuras de diferentes antigüedades y estilos constructivos. En el sector central está emplazada la casa principal en forma de U, la "casa de verano", un sector de oficinas, dependencias de los mayordomos, patio y galerías. En este mismo cuadro se ubica una pileta de forma ovalada y dos aljibes. Algo más alejadas se encuentran otras edificaciones, como la carnicería, galpones y cochera. Separados por alambrado y un cerco vivo, a unos $80 \mathrm{~m}$ de la casa principal, se ubican dos estructuras que corresponden a la vivienda de los peones, cocina y galpón de trabajo (García Lerena, 2018).

Con el objetivo de caracterizar las prácticas cotidianas de distintos sectores que habitaron y transitaron por esta estancia, la conformación de los espacios de esta unidad productiva e indagar los cambios que experimentaron a lo largo del tiempo, entre los años 2016 y 2018, se realizó un relevamiento in situ de las estructuras, prospecciones y excavaciones sistemáticas en el sector interpretado como basurero rural a cielo abierto y una amplia búsqueda y análisis de fuentes inéditas que fue articulada con la información arqueológica. En la actualidad, sólo parte de las edificaciones de todo el sector del casco continúa en uso; la antigua casa del mayordomo y la "casa de verano" fueron reformadas y acondicionadas. También se utiliza el galpón moderno para guardar algunas maquinarias. El resto de las construcciones de la estancia se encuentran cerradas y en desuso desde hace años, evidenciando distintos estados de conservación.

En particular, el sector objeto de este trabajo es la antigua vivienda de los peones, una estructura de material, con techo de chapa a dos aguas con seis habitaciones internas y un pasillo central. Se eligió esta estructura para utilizar la técnica SfM-MVS, que será desarrollada a continuación.

\section{Métodos}

El flujo de trabajo (Figura 2) para este caso en particular ha sido la toma de fotografías en una trayectoria circular con cámara en mano. Se registraron 70 fotografías de la vivienda de los peones de "Primera Estancia" en una circunferencia completa y 19 fotografías extras para relevar detalles y evitar la oclusión visual por árboles cercanos a la estructura. Para ello se utilizó una cámara Nikon D5100 con un sensor de $16 \mathrm{Mpx}$ y una lente fija de $50 \mathrm{~mm}^{1}$. La distancia entre la lente y la estructura fue en promedio de $10,8 \mathrm{~m}$. Las fotografías fueron capturadas en formato *.jpg, con tamaño de 4928 x 3264 píxeles. La resolución espacial promedio del modelo, es decir el tamaño del píxel, fue de 2,12 mm.

El software empleado para todo el flujo de trabajo fue Agisoft Photoscan professional (versión 1.4.0). Luego de cargar las fotografías se enmascaró la estructura en cada una de ellas manualmente, con el fin de reservarla; esto permitió excluir puntos que no se correspondían con el objeto a modelar, en particular aquellos con movimiento (vegetación y sombras) o sin textura (por ejemplo, el cielo) para mejorar el proceso de alineación de las fotografías (de Reu et al., 2013).

1 Las lentes con focales fijas son las que generan menor distorsión, aberraciones cromáticas y falta de nitidez. Se recomienda utilizar lentes fijas dentro el rango de 35 a 105 mm (Mosbrucker, Major, Spicer y Pitlick, 2017). 


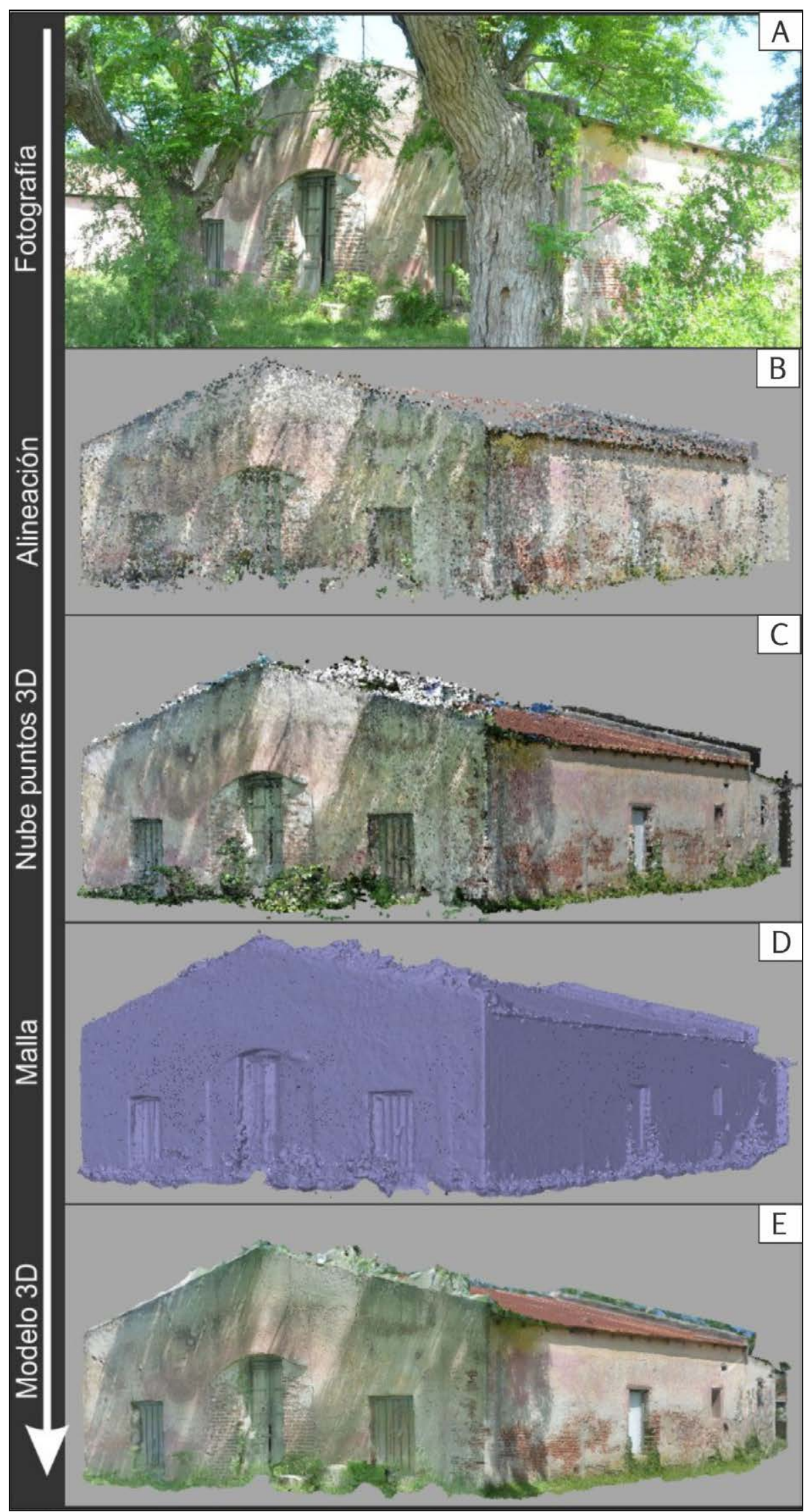

Figura 2. A) Ejemplo de fotografía de la vivienda de los peones. B) Alineación de puntos. C) Nube de puntos densa 3D. D) Malla. E) Modelo 3D con textura. 
Luego de preparar las fotografías el procesamiento continuó con un flujo semiautomático que consistió en la alineación de las mismas identificando puntos clave entre pares de fotos para generar una nube de puntos 3D. A partir de esta nube se realizó una malla -superficie generada mediante triangulación-, utilizando como vértices los puntos de la nube densa. Sobre esta nueva superficie fue posible agregar textura a partir de las fotografías para darle al modelo una imagen fotorrealista. Para lograr las mediciones en el post-procesamiento es necesario escalar o georreferenciar el modelo; para lo cual se debe contar con una precisión muy alta en la determinación de las coordenadas de los puntos de control. Las herramientas recomendadas para relevar estos puntos serían Estación total (ET) o GPS diferencial (dGPS). Como para el objetivo de este trabajo bastaba con un modelo escalado, se optó por una cinta métrica de 50 m milimetrada, que tiene la misma resolución que una ET y mayor resolución que un dGPS, con un costo sustancialmente más bajo y un manejo más sencillo.

Para el escalado se tomaron medidas de las dimensiones de los lados externos de la estructura a relevar. También, se tomaron medidas auxiliares de aberturas para evaluar la precisión del modelo digital y también para comprobar las longitudes en planos verticales. Estos datos se cargaron en el software y se generaron escalas virtuales; de esta forma fue posible determinar el error del modelo. En nuestro caso de estudio, utilizando seis escalas virtuales en diferentes orientaciones, el error fue de $2,6 \mathrm{~cm}$.

\section{Resultados}

Los parámetros utilizados para la producción del modelo mediante SfM-MVS han sido compilados en la Tabla 1, allí también se indican las propiedades del modelo obtenido.

El producto final fue un modelo 3D fotorrealista, que contiene el registro integral de la información gráfica y presenta el estado actual del edificio permitiendo saber su situación, forma y estructura en un momento determinado. El modelo escalado permitió tomar otras mediciones en gabinete. Esta posibilidad redujo el tiempo de registro de datos en

\begin{tabular}{|c|c|c|}
\hline \multirow{2}{*}{ Fotografías } & Número & Modelo 3D \\
\hline & Distancia promedio $(\mathrm{m})$ & 89 \\
& Resolución $(\mathrm{mm})$ & 10,8 \\
& Alineación & 2,12 \\
\hline \multirow{2}{*}{ Puntos clave } & Precisión & 110.120 \\
& Key point limit & Alta \\
& Tie point limit & 400.000 \\
& Cantidad de puntos & 10.000 \\
\hline \multirow{3}{*}{ Nube de puntos densa } & Calidad & 15.626 .638 \\
& Filtrado de profundidad & Alta \\
& Caras & Moderado \\
\hline Malla & Vértices & 1.045 .280 \\
& Tipo de superficie & 525.274 \\
& Interpolación & Alta \\
& Tamaño & Habilitado \\
\hline
\end{tabular}

Tabla 1. Parámetros utilizados para la producción del modelo virtual 3D de la vivienda de peones. 
el campo y permitió volver a cotejar o corroborar datos faltantes en el laboratorio. Otro aspecto a destacar es que, a diferencia de las fotografías $2 \mathrm{D}$, las visuales obtenidas desde el modelo no presentan oclusión por parte de vegetación u otras edificaciones. Esto es posible ya que al enmascarar cada una de las fotos se genera un entorno sin obstáculos para el análisis. En particular, resultó de importancia en el caso de los árboles que cubren un sector relevante de la estructura, mientras que en la visual del modelo 3D estos árboles fueron eliminados utilizando las máscaras (comparar Figura 2A y 2E). Los modelos 3D realizados con máscaras permiten desechar gran parte de los elementos circundantes que no son de interés. Esto permite obtener visuales con menos obstáculos que las fotografías estándar tomadas desde la misma perspectiva.

Como producto final del procesamiento el modelo se exportó con formato *.obj y se alojó en https://skfb.ly/6THu6 ${ }^{2}$, lo cual permite su visualización en un entorno 3D en cualquier dispositivo con acceso a internet.

\section{Análisis espacial de las patologías}

La vivienda de los peones presenta diversas patologías y deterioros debido a su falta de mantenimiento, a factores físicos y a agentes biológicos, los que afectan con distinta gravedad la estructura. Para su relevamiento se utilizó una ficha con la especificación de los siguientes parámetros: $\mathrm{N}^{\circ}$ de ficha, edificio, localización del edificio, tipo de patología (física, química, físico-química, mecánica), patología (grietas, fisuras, desprendimientos; insectos xilófagos, humedad, etc.), localización de la patología, ubicación en la planta del edificio (croquis 2D), descripción, fotografía de detalle, mapeo de la patología sobre las caras del edificio (realizado a posteriori del modelo, sobre el ortomosaico de cada una de las caras; ejemplo en la Figura 3).

Se identificaron numerosas y profundas grietas, fisuras y desprendimiento de revoque en grandes superficies, que exponen los ladrillos interiores, en particular en la cara oeste. También se observaron pátinas verdes producto del biodeterioro (algas), en la cara este. Otras patologías importantes las constituyen los insectos xilófagos que debilitan y destruyen la madera (presente en puertas, ventanas y tirantes de techos), nidos de avispas en los tirantes del techo, los roedores cavadores que realizan sus cuevas al interior de la estructura y los murciélagos que la habitan. Estos últimos dificultan las tareas en el interior de la vivienda, a la vez que la acumulación de sus excrementos puede tener consecuencias químicas sobre los materiales que afecta.

El registro de estas patologías fue realizado sobre el ortomosaico, exportado del modelo generado por el Agisoft, en el programa QGIS (versión 3.6). Este mapeo y digitalización, sobre el ortomosaico, de cada una de las patologías permitió la delimitación de polígonos, que fueron escalados, referenciados y ortorrectificados. En la Figura 3 se presenta el registro de las patologías mapeadas sobre la cara este de la estructura. La ventaja que presenta realizar este registro sobre el modelo o sobre el ortomosaico, en comparación con las fotografías de alta calidad, es que estas últimas presentan deformación, que es corregida en el modelo y por consiguiente en el ortomosaico, aportando una mayor precisión incluso frente a fotografías ortorrectificadas con puntos de control (de Reu et al., 2013). A su vez, el mapeo de las patologías en conjunto permite establecer asociaciones espaciales entre ellas, evidenciar posibles patrones y su relación con factores externos (por ejemplo, orientación, exposición solar o vientos predominantes).

2 Es necesario aclarar que las partes faltantes que se observan en el techado de la estructura se deben a que las fotografías fueron tomadas por un usuario de pie. Esto podría corregirse mediante el uso de otro tipo de plataformas, como un dron o una pértiga, la segunda opción con un costo más accesible que la primera. 


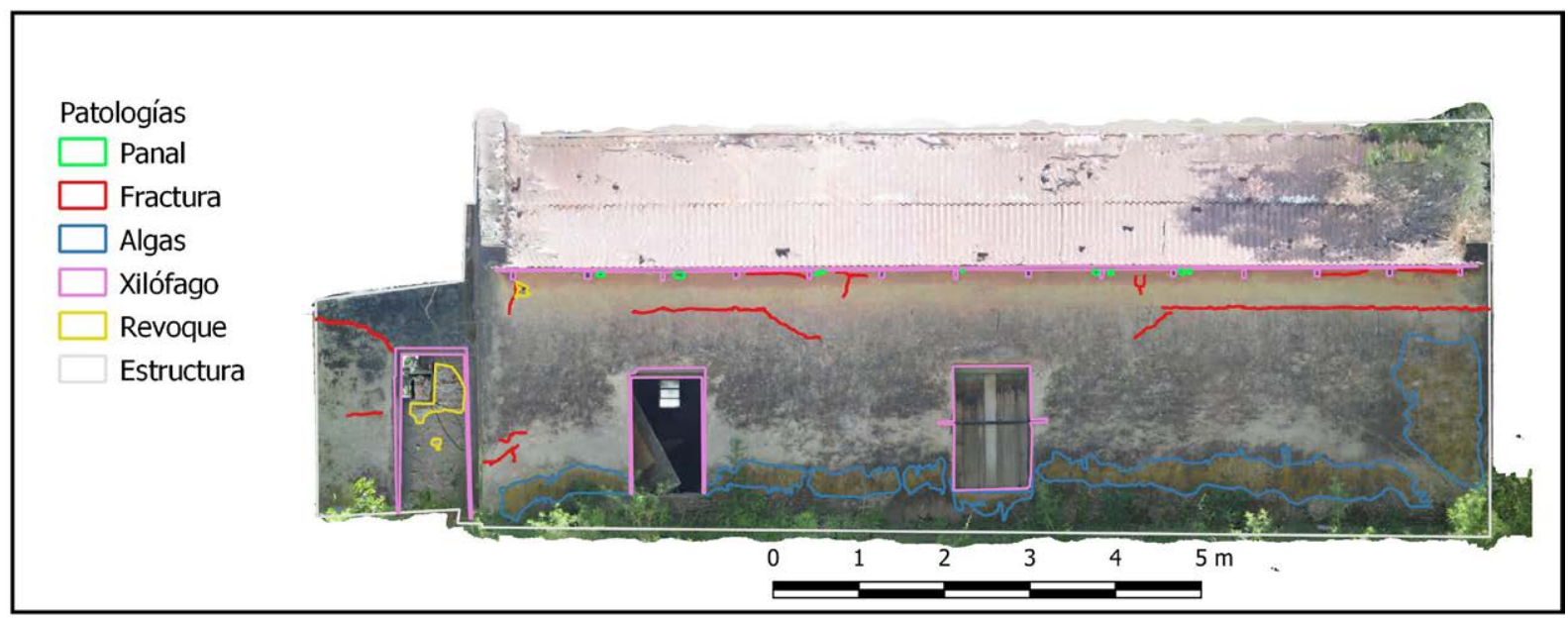

Figura 3. Mapeo de las patologías sobre el ortomosaico generado a partir del modelo (cara este de la estructura).

\section{Potencialidades y limitaciones de la técnica}

La potencialidad del SfM-MVS radica en la reducción del tiempo de toma de datos, su bajo costo, alta resolución y que constituye una técnica preferible a otras tradicionales, más lentas y con resolución más baja (Green, Bevan y Shapland, 2014). A su vez, permiten un relevamiento in situ, no destructivo, que no requiere ni equipamientos específicos ni técnicas complejas. También es valorable la posibilidad que brinda de obtener reconstrucciones con gran detalle y precisión, que permiten realizar mediciones lineales, superficiales y volumétricas en gabinete. Esto es de gran importancia al trabajar en propiedades privadas, a la vez que permite abordar las estructuras en forma fácil y rápida en situaciones dificultosas para la obtención de permisos.

A partir del modelo generado, es posible registrar, documentar y archivar bienes patrimoniales, en este caso la vivienda de los peones de una estancia ganadera; además, permite conservar un relevamiento de las patologías que los afectan. Una posible limitación de la técnica descripta es el relevamiento de elementos paisajísticos como son las arboledas de parques y jardines que constituyen un aspecto importante del patrimonio local en general y de esta estancia en particular (García Lerena, Doumecq, Stampella y Pochettino, 2018). Esta limitación se debe a la movilidad del follaje por el viento y por la complejidad de la estructura vegetal (Carrivick, Smith y Quincey, 2016; Willis et al., 2016).

En el caso de esta estancia, si bien es relativamente cercana a los centros poblados, los caminos vecinales de acceso se presentan como de difícil acceso en épocas de lluvias, a lo que se suma la coordinación que debe llevarse a cabo con los propietarios de la estancia, que no residen en ella a tiempo completo. De esta manera, dos investigadores con el equipamiento necesario pudieron realizar el relevamiento de todas las estructuras del casco de la estancia en una jornada de trabajo, generándose un croquis 2D y la adquisición de las imágenes de las distintas estructuras para el modelado posterior en gabinete.

Primera Estancia, por sus características señaladas previamente, es significativa para el patrimonio local y relevante en las valoraciones de los pobladores locales. Por ello esta técnica es especialmente atractiva, ya que resulta adecuada para documentar y registrar elementos patrimoniales, aportando imágenes con colores y dimensiones reales, a partir de las cuales se puede obtener información detallada en gabinete. La 
posibilidad de trabajar con el modelo en el gabinete permite una retroalimentación entre interpretaciones y observaciones o mediciones.

El trabajo de las patologías y deterioros se realizó en forma preliminar en el terreno y fue desarrollado en profundidad en gabinete a partir del modelo. Esto disminuyó el tiempo de toma de datos en campo y permitió obtener un estado de la estructura y las patologías y deterioros que la afectan, para avanzar sobre sus posibles causas y buscar asesoramiento para su preservación y rehabilitación.

Diversos autores han señalado las potencialidades que tiene el SfM-MVS para la divulgación y socialización de resultados de investigación (Díaz Gómez et al., 2015). En este sentido, el sitio Estancia Bertón (partido de Magdalena), una estancia cercana abordada en trabajos previos de investigación, presentaba una estructura significativa, un "rancho" confeccionado a dos aguas con techo de paja que reunía materiales y técnicas constructivas originales ${ }^{3}$ (García Lerena, 2016). Esta estructura se encontraba en un estado de conservación muy deficiente cuando se realizaron las tareas de campo en 2012. Finalmente, debido a la imposibilidad de su cuidado por sus propietarios y la inacción de esferas municipales, se derrumbó en el año 2016, constituyendo una pérdida irreparable para el patrimonio local. Esta situación, en donde en pocos años desde su investigación el rancho de mayor antigüedad y autenticidad para un período histórico se destruyó, nos habilita a reflexionar sobre las potencialidades de esta técnica. Si bien un modelo, por más calidad y realismo que tenga, nunca va a reemplazar la realidad, contar con este tipo de representación hubiera permitido su salvaguarda en formato digital y habilitado su reestudio con nuevos objetivos o por nuevos investigadores.

Como producto final del procesamiento es posible exportar la nube de puntos, la malla o el modelo con la textura en distintos formatos (*.obj, *.stl, *.pdf). Para la difusión y socialización del producto final, el modelo realizado puede ser compartido vía *.pdf y/o subido a repositorios de modelos tridimensionales en la nube (Champion y Rahaman, 2020). Las ventajas que brinda la técnica para compartirse por diferentes vías, en particular la web (Morita y Bilmes, 2018), amplía las posibilidades para que el patrimonio local se conozca y se genere responsabilidad social en su cuidado y puesta en valor.

\section{Perspectivas a futuro}

Una forma de potenciar y complementar nuestro trabajo sería realizar relevamientos periódicos de la estructura y evaluar las modificaciones entre los sucesivos modelos a lo largo del tiempo, para comprobar la evolución temporal del bien patrimonial y evaluar el posible avance de las patologías y deterioros. Otro aspecto que se pretende abordar es el uso de alguna plataforma para sacar fotografías de altura y mejorar la composición del modelo, en particular el techo. Asimismo, se prevé complementar este modelado con el de otras estructuras de la estancia no modeladas hasta el momento.

Por otro lado, el modelo podría ser georreferenciado en el campo, y exportarse en formato *.kmz para su vista en Google Earth, lo que permitiría contextualizar su localización y entorno. Para ello es necesario contar con puntos de control con coordenadas conocidas obtenidos mediante ET o dGPS. Los repositorios en la nube

3 Las paredes, denominadas quinchadas, eran de postes de madera, atados con tientos de cuero crudo y paneles con un centro de paja gruesa recubiertos de barro. Este rancho, es ejemplo de un tipo de construcción muy característico y abundante en el pasado de la región de la pampa húmeda, de los que hoy en día se conservan pocos y en mal estado. 
y los *.kmz se configuran no solamente como insumo para análisis posteriores, sino también como una excelente vía de socialización y difusión.

Por lo expuesto, consideramos que esta técnica es una importante herramienta para la socialización de la información en la comunidad académica y posibilita de forma sencilla y con bajo costo una divulgación de los modelos por diferentes vías digitales, los cuales pueden resultar accesibles, además, para la comunidad local (Morita y Bilmes, 2018; Sánchez Chacón y Shimabukuro Shimabukuro, 2015). Para ello, consideramos que se deben generar planes de manejo y gestión del patrimonio desde las distintas esferas de responsabilidad, de acuerdo a los intereses locales y definidos en la interacción comunidad y diferentes organismos oficiales (Paleo et al., 2015). De esta manera, todo bien es potencialmente patrimonializable, en la medida que adquiere un valor representativo de la historia, identidad y cultura de una comunidad, y es ésta quien debe realizar el proceso de patrimonialización. Sin embargo, la conservación adecuada y puesta en valor de los bienes patrimoniales no debería recaer sólo en voluntades particulares, sino que requieren de la participación activa de distintos ámbitos de gestión estatal (García Lerena, 2016). Herramientas como SfM-MVS posibilitarían otorgar mayor difusión al patrimonio local, su apropiación por la comunidad y el diseño de estrategias para su uso sustentable.

Una propuesta a futuro que consideramos prometedora consiste en, previo consentimiento de los propietarios, realizar un relevamiento virtual de las edificaciones de las estancias significativas de la zona para confeccionar un atlas y/o un recorrido virtual desde diferentes soportes. Esta propuesta debería motorizarse en forma articulada entre miembros de la comunidad y asociaciones locales, Municipalidades de Magdalena y Punta Indio, la Universidad Nacional de La Plata y la UNESCO.

\section{Agradecimientos}

Esta investigación se realizó en el marco del proyecto "Investigaciones arqueológicas en los Partidos de Magdalena, Punta Indio y zona costera de Berisso, Provincia de Buenos Aires. Dinámicas y procesos", dirigido por María Clara Paleo. Queremos expresar nuestro agradecimiento a los dueños de las estancias que nos permitieron desarrollar nuestras investigaciones en sus propiedades. A los evaluadores del manuscrito, quienes con sus comentarios y sugerencias contribuyeron a mejorarlo sustancialmente. 


\section{Referencias citadas}

» Aparicio Resco, P., Carmona Barrero, J. D., Fernández Díaz, M. y Martín Serrano, P. M. (2014). Fotogrametría involuntaria: rescatando información geométrica en 3D de fotografías de archivo. Virtual Archaeology Review, 5(10), 11-20. https://doi.org/10.4995/var.2014.4205.

» Benavides López, J. A., Jiménez, G. A., Romero, M. S., García, E. A., Martín, S. F., Medina, A. L. y Guerrero, J. A. E. (2016). 3D modelling in archaeology: the application of Structure from Motion methods to the study of the megalithic necropolis of Panoria (Granada, Spain). Journal of Archaeological Science: Reports, 10, 495-506. https://doi.org/10.1016/ j.jasrep.2016.11.022

» Carrivick J. L., Smith, M. W. y Quincey, D. J. (2016). Structure from Motion in the geo. West Sussex: Wiley Blackwell.

» Champion, E. y Rahaman, H. (2020). Survey of 3D Digital Heritage Repositories and Platforms. Virtual Archaeology Review, 11, 1-15. https://doi.org/10.4995/var.2020.13226.

» Charquero Ballester, A. (2016). Práctica y usos de la fotogrametría digital en arqueología. DAMA, 1, 139-157. http://dx.doi.org/10.14198/dama.2016.1.10

» De Reu, J., De Smedt, P., Herremans, D., Van Meirvenne, M., Laloo, P. y De Clercq, W. (2014). On introducing an image-based 3D reconstruction method in archaeological excavation practice. Journal of Archaeological Science, 41, 251-262. http://doi.org/10.1016/j.jas.2013.08.020

» De Reu, J., Plets, G., Verhoeven, G., De Smedt, P., Bats, M., Cherretté, B., De Maeyer, W., Deconynck, J., Herremans, D., Laloo, P., Van Meirvenne, M. y De Clercq, W. (2013). Towards a three-dimensional cost-effective registration of the archaeological heritage. Journal of Archaeological Science, 40(2), 1108-1121. http://doi.org/10.1016/j.jas.2012.08.040

» Díaz Gómez F., Jiménez Peiró, J., Barreda Benavent, A., Asensi Recuenco, B. y Hervás, J. (2015). Modelado 3D para la generación de patrimonio virtual. Virtual Archaeology Review, 8(12), 29-37. https://doi.org/10.4995/var.2015.4150.

» Evin, A., Souter, T., Hulme-Beaman, A., Ameen, C., Allen, R., Viacava, P., Larson, G., Cucchi, T. y Dobney, K. (2016). The use of close-range photogrammetry in zooarchaeology: creating accurate 3D models of wolf crania to study dog domestication. Journal of Archaeological Science, 9, 87-93. http://10.1016/j.jasrep.2016.06.028

» García Lerena, M. S. (2016). Patrimonio e historia del sitio “Estancia Bertón”. Un rancho pampeano a la vera del camino (Partido de Magdalena, Buenos Aires, Argentina). Revista Canto Rodado, 11, 79-106.

» García Lerena, M. S. (2018). Historia de un paisaje pampeano: orden, segmentación y diferenciación en "Primera Estancia" (Magdalena, Buenos Aires, Argentina). Revista del Museo de la Plata, 3(1), 148-163. https://doi.org/10.24215/25456377e056

» García Lerena, M. S., Doumecq, M. B, Stampella, P. C. y Pochettino, M. L. (2018). Las raíces del paisaje: los árboles en la construcción de las estancias de la región pampeana argentina. En M. Alcántara, M. García Montero y F. Sánchez López (Coord.), Ciencias y medio ambiente: $56^{\circ}$ Congreso Internacional de Americanistas (pp. 115-132). Salamanca: Ediciones Universidad de Salamanca.

» Green, S., Bevan, A. y Shapland, M. (2014). A comparative assessment of structure from motion methods for archaeological research. Journal of Archaeological Science, 46, 173-181. https://doi.org/10.1016/j.jas.2014.02.030.

» Hatzopoulos, J. N., Stefanakis, D., Georgopoulos, A., Tapinaki, S., Pantelis, V. y Liritzis, I. (2017). Use of various surveying technologies to 3D digital mapping and modelling of cultural heritage structures for maintenance and restoration purposes: The Tholos in Delphi, Greece. Mediterranean Archaeology and Archaeometry, 17, 311-336. https://doi. org/10.5281/zenodo. 1048937 
» Lorenzo, G., López, L. y Flores, M. (2016). Del dibujo a mano alzada a la Fotogrametría digital: explorando nuevas técnicas de registro del Patrimonio arqueológico. En A. Laguens, M. Bonnin y B. Marconetto (comps.), Libro de resumes del XIX Congreso Nacional de Arqueología Argentina (pp. 3150-3152). Córdoba: IDACOR-CONICET, UNC.

» Maté González, M., Yravedra, J., González-Aguilera, D., Palomeque-González, J. y DomínguezRodrigo, M. (2015). Micro-photogrammetric characterization of cut marks on bones. Journal of Archaeological Science, 62, 128-142. https://doi.org/10.1016/j.jas.2015.08.006

» Morita, M. y Bilmes, G. (2018). Applications of low-cost 3D imaging techniques for the documentation of heritage objects. Optica Pura y Aplicada, 51, 1-11. http://dx.doi.org/10.7149/ OPA.51.2.50026.

» Mosbrucker, A. R., Major, J. J., Spicer, K. R. y Pitlick, J. (2017). Camera system considerations for geomorphic applications of SfM photogrammetry. Earth Surface Processes and Landforms, 986, 969-986. https://doi.org/10.1002/esp.4066.

»Paleo, M. C., Pérez Meroni, T. M., Ghiani Echenique, N., Uvietta, A., Day Pilaría, F. y García Lerena, M. S. (2015). Las Áreas Protegidas como escenario para el manejo de los bienes culturales-naturales y su patrimonialización. El caso del Parque Costero del Sur. En 0. Palacios, C. Vázquez y N. Ciarlo (Eds.), Patrimonio cultural: la gestión, el arte, la arqueología y las ciencias exactas aplicadas. Actas IV Jornadas Nacionales para el estudio de Bienes Culturales (pp. 155-165). Buenos Aires: Ediciones Nuevos Tiempos.

» Paleo, M. C., García Lerena, M. S., Stampella, P. C., Doumecq, M. B. y Pochettino, M. L. (2016). Las estancias y sus árboles. En La construcción del paisaje del litoral rioplatense (Tomo 2). La Plata: Editorial Universitaria de La Plata y Xitix S. A.

» Pereira Uzal, J.M. (2013). Modelado 3D en Patrimonio Cultural por Técnicas de Structure from Motion. Ph Investigación, 1, 77-87.

» Pierdicca, R., Frontoni, E., Malinverni, E. S., Colosi, F. y Orazi, R. (2016). Virtual reconstruction of archeological heritage using a combination of photogrammetric techniques: Huaca Arco Iris, Chan Chan, Peru. Digital Applications in Archaeology and Cultural Heritage, 3, 80-90. http:// doi.org/10.1016/j.daach.2016.06.002.

» Sánchez Chacón, N. y Shimabukuro, Shimabukuro P. (2015). 'Structure from motion' Alternativa de bajo costo para el registro del patrimonio edificado. Devenir, 2(4), 150-178.

» Scopigno, R., Callieri, M., Cignoni, P., Corsini, M., Dellepiane, M., Ponchio, F. y Ranzuglia, G. (2011). 3D Models for Cultural Heritage: Beyond Plain Visualization. Computer, 44(7), 48-55. http://doi.org/10.1109/mc.2011.196

»Vázquez, F. y Díaz Pais, E. (2014). Arqueología virtual en una estancia colonial argentina. Virtual Archaeology Review, 5(10), 6-10. https://doi.org/10.4995/var.2014.4204.

» Verhoeven, G., Doneus, M., Briese, C. y Vermeulen, F. (2012). Mapping by matching: A computer vision-based approach to fast and accurate georeferencing of archaeological aerial photographs. Journal of Archaeological Science, 39(7), 2060-2070. http://doi.org/10.1016/j. jas.2012.02.022

»Willis, M. D., Koenig, C. W., Black, S. L. y Castaneda, A. (2016). Archeological 3D mapping: the structure from motion revolution. Taking Photographs for SfM. Journal of Texas Archeology and History, 3, 1-36. 